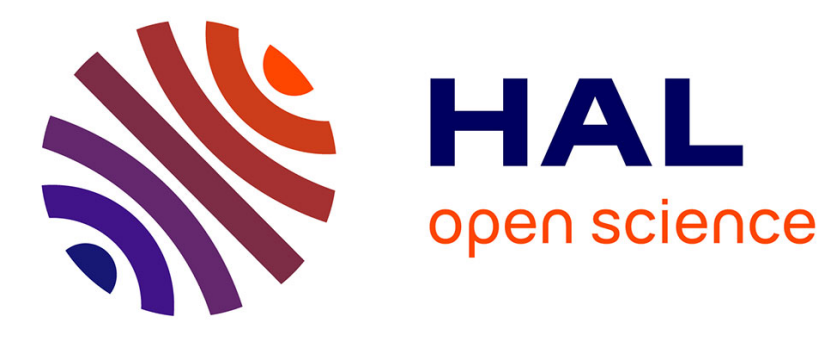

\title{
UAV Trajectory Planning for Delay Tolerant Communications
}

Daniel Bonilla Licea, Moisés Bonilla, Mounir Ghogho, Michel Malabre

\section{To cite this version:}

Daniel Bonilla Licea, Moisés Bonilla, Mounir Ghogho, Michel Malabre. UAV Trajectory Planning for Delay Tolerant Communications. 58th IEEE Conference on Decision and Control (CDC 2019), Dec 2019, Nice, France. 10.1109/CDC40024.2019.9030162 . hal-02266464

\section{HAL Id: hal-02266464 https://hal.science/hal-02266464}

Submitted on 26 Nov 2020

HAL is a multi-disciplinary open access archive for the deposit and dissemination of scientific research documents, whether they are published or not. The documents may come from teaching and research institutions in France or abroad, or from public or private research centers.
L'archive ouverte pluridisciplinaire HAL, est destinée au dépôt et à la diffusion de documents scientifiques de niveau recherche, publiés ou non, émanant des établissements d'enseignement et de recherche français ou étrangers, des laboratoires publics ou privés. 


\title{
UAV Trajectory Planning for Delay Tolerant Communications
}

\author{
Daniel Bonilla Licea, M. Bonilla, Mounir Ghogho and M. Malabre
}

\begin{abstract}
In this paper, we address the problem of optimizing a communication-aware trajectory for a quadrotor that must transfer periodically (with fixed period $T$ ) a maximum amount of data from a source node (SN) to a destination node (DN). The communications aspect is mathematically stated by linking the bit rate to the channel capacity concept from information theory. The trajectory is optimized using a parametric approach using Fourier series in order to reduce the computational load of the optimization process. We show that the proposed trajectory results in a large increase of the amount of transferred data, and can be easily tracked by the quadrotor.
\end{abstract}

Index Terms - Trajectory planning, data ferry, delay tolerant communications.

\section{NOTATION}

A zero dimension subspace is denoted by $\{0\}$, and the identity operator is denoted by $\mathrm{I}$, or $\mathrm{I}_{n}$ when its size, $n \times n$, is specified. For a full row (column) rank matrix $X \in \mathbb{R}^{n \times m}$, its right (left) inverses are denoted by $X^{r}\left(X^{\ell}\right)$, i.e. $X X^{r}=\mathrm{I}_{n}\left(X^{\ell} X=\mathrm{I}_{m}\right)$. If $X$ is square and invertible, its inverse is denoted by $X^{-1} \cdot x^{\mathrm{T}}$ stands for the transpose of the matrix (or vector) $x$. s stands for the complex variable of Laplace transform. Given a pair of mappings $(A, B)$, such that $A: \mathbb{R}^{n} \rightarrow \mathbb{R}^{n}$ and $B: \mathbb{R}^{m} \rightarrow \mathbb{R}^{n}, \mathcal{C}_{(A, B)}$ stands for the controllability matrix $\mathcal{C}_{(A, B)}=\left[\begin{array}{lllll}B & A B & \cdots & A^{(n-1)} B\end{array}\right]$. $\Psi_{m}(\xi) \in \mathbb{R}^{n \cdot m \times m}[\xi]$ stands for: $\left[\begin{array}{llll}\mathrm{I}_{m} \xi & \mathrm{I}_{m} \xi^{2} & \ldots & \mathrm{I}_{m} \xi^{n}\end{array}\right]^{T}$.

Further, $\mathrm{c}(\vartheta)$ and $\mathrm{s}(\vartheta)$ stand for cosine and sine of a given angle $\vartheta$, respectively; and $s^{-1}(\vartheta)$ stands for $\arcsin (\vartheta)$. The ceiling and the floor functions are denoted as $\lceil\cdot\rceil$ and $\lfloor\cdot\rfloor$ respectively. $\hat{x}$ stands for the estimate of the variable $x ; \mathbb{E}[\cdot]$ stands for the statistical expectation operator.

A vector function $f(\varepsilon, t) \in \mathbb{R}^{n}$ is said to be $\mathcal{O}(\varepsilon)$ over an interval $\left[t_{1}, t_{2}\right]$ if there exist positive constants $K$ and $\varepsilon^{*}$ such that $\|f(\varepsilon, t)\| \leq K \varepsilon$ for all $\varepsilon \in\left[0, \varepsilon^{*}\right]$ and for all $t \in\left[t_{1}, t_{2}\right]$, see [4].

\section{INTRODUCTION}

The concept of communication-aware trajectory planning for robots has recently attracted an increasing amount of attention within the robotics, control and communications communities. This interdisciplinary research field aims at improving robots' wireless communications performance by controlling their position [16]-[18]. In this context, various problems have been considered: the utilisation of a robot to physically transfer data from the source to the destination [16]; the optimization of a robot's trajectory taking into

Daniel Bonilla Licea is with the International University of Rabat, Morocco, e-mail: daniel.bonilla-licea@uir.ac.ma.

M. Bonilla is with the CINVESTAV-IPN, UMI 3575 CINVESTAV-CNRS, Mexico, e-mail: mbonilla@cinvestav.mx.

Mounir Ghogho is with the International University of Rabat, Morocco and the University of Leeds, UK, e-mail: m. ghoghodieee. org.

M. Malabre is with CNRS, LS2N, UMR 6004, Nantes, France, e-mail: Michel. Malabredls2n.fr. account motion and communications energy [17]; the minimization of energy consumption of a wireless sensor network using autonomous mobile robots [19].

In this paper, we consider the following scenario. A battery-powered source node (SN) has to periodically transmit data to a destination node (DN), and the data transfer is delay tolerant. The distance between $\mathrm{SN}$ and DN is assumed too large for a direct communication as this would require too much energy. The SN may be equipped with an energy harvesting system to recharge its battery, but this recharging is assumed insufficient to sustain direct communication with the DN. A quadrotor acts as a dedicated data ferry [16] between the $\mathrm{SN}$ and the DN, i.e. it collects data from the $\mathrm{SN}$ and then moves towards the DN to deliver the data; this process is performed periodically. In this paper, we design the quadrotor's trajectory to maximize the amount of data transferred from the SN to the DN in each period, while taking into account a realistic wireless communication model as well as a realistic quadrotor dynamical model. To the authors' knowledge, this particular communication-aware trajectory problem has not been addressed before.

This paper is organized as follows. In section II we first describe the wireless channel model and then the quadrotor dynamical model. In section III, we present a feedback linearization method for the quadrotor's model. In section IV, we state the trajectory optimization problem. The solution to this problem is described in section V. Conclusions are drawn in section VI.

\section{SYSTEM MODEL}

\section{A. Communications System Model}

Let us denote the position of the $\mathrm{SN}$ and $\mathrm{DN}$ in the $\mathrm{x}-\mathrm{y}$ plane as $\mathbf{p}_{0} \in \mathbb{R}^{2}$ and $\mathbf{p}_{1} \in \mathbb{R}^{2}$ respectively. For mathematical convenience and without loss of generality, we set $\mathbf{p}_{0}=\left[\begin{array}{ll}D / 2 & 0\end{array}\right]^{\mathrm{T}}$ and $\mathbf{p}_{1}=\left[\begin{array}{ll}-D / 2 & 0\end{array}\right]^{\mathrm{T}}$.

We assume that the heights of the SN and DN antennas are the same and larger than those of most of the nearby obstacles. In addition, the quadrotor is assumed to operate on the horizontal plane defined by the positions of the SN and DN antennas. The position of the quadrotor on this plane is denoted by $\mathbf{p}(t) \in \mathbb{R}^{\mathrm{T}}$, namely: $\mathbf{p}(t)=\left[\begin{array}{ll}\mathrm{x}(t) & \mathrm{y}(t)\end{array}\right]^{\mathrm{T}}$. Therefore, we assume that here is a line of sight between the quadrotor and both the SN and the DN. Hence, the wireless channel can be modelled as an additive white Gaussian noise (AWGN) channel with free-space pathloss only [13]. The signal-to-noise ratio (SNR) of the signal received by the DN from the quadrotor (i.e., the ratio of the power of the signal received by the $\mathrm{DN}$ from the quadrotor to the power of the 
noise generated at the DN's receiver) can then be expressed as:

$$
\Gamma\left(\mathbf{p}(t), \mathbf{p}_{1}\right)=P /\left(\left\|\mathbf{p}_{1}-\mathbf{p}(t)\right\|_{2}^{2} \sigma^{2}\right),
$$

where $\sigma^{2}$ is the noise power at the DN's receiver and $P$ is the power of the signal transmitted by the quadrotor. We assume that the SN and the quadrotor have the same transmit power, $P$, and that the noise at the quadrotor's and DN's receivers have the same power, $\sigma^{2}$. The SNR of the signal received by the quadrotor from the $\mathrm{SN}$ is thus given by $\Gamma\left(\mathbf{p}(t), \mathbf{p}_{0}\right)$.

Finally, the quadrotor's communication system operates on a semi-duplex mode ${ }^{1}$. So, we introduce the binary function $S W(t)$ that indicates the operational mode of the quadrotor's communications system at time $t$. If $S W(t)=1(S W(t)=$ 0 ) the quadrotor's communication system operates as a transmitter (receiver) at time instant $t$.

\section{B. Quadrotor Dynamical Model}

The dynamical model of the quadrotor considered in this paper is given by (see Section 5 of [9] for details):

$$
\begin{array}{ll}
\mathrm{d}^{2} \mathrm{x} / \mathrm{d} t^{2}=-g\left(\theta-\bar{q}_{\mathrm{x}}\right), & \mathrm{d}^{2} \theta / \mathrm{d} t^{2}=u_{\mathrm{x}} / \mathcal{I}_{\mathrm{y}}+q_{\theta}, \\
\mathrm{d}^{2} \mathrm{y} / \mathrm{d} t^{2}=g\left(\phi+\bar{q}_{\mathrm{y}}\right), & \mathrm{d}^{2} \phi / \mathrm{d} t^{2}=u_{\mathrm{y}} / \mathcal{I}_{\mathrm{x}}+q_{\phi}, \\
\mathrm{d}^{2} \mathrm{z} / \mathrm{d} t^{2}=u_{\mathrm{z}} / M+g+q_{\mathrm{z}}, & \mathrm{d}^{2} \psi / \mathrm{d} t^{2}=u_{\psi} / \mathcal{I}_{\mathrm{z}}+q_{\psi},
\end{array}
$$

where $M$ is the quadrotor's total mass, the moments of inertia about axis $o x$, oy and $o z \operatorname{are}^{2} \mathcal{I}_{x}, \mathcal{I}_{y}$ and $\mathcal{I}_{z}$, the distance of each rotor with respect to the centre of gravity of the quadrotor is $L$. The motion of the quadrotor is described with respect to a fixed orthogonal axis set (oxyz), where $o z$ points vertically up along the gravity vector $\left[\begin{array}{ccc}0 & 0 & g\end{array}\right]^{T}$. The origin $o$ is located at the same height as the SN and DN antennas. The coordinates $x, y$ and $z$ refer to the position of the centre of gravity of the quadrotor in the space where $z$ is its altitude. Note that $\mathbf{p}(t)$ in section II-A is given by $[x, y]^{\mathrm{T}}$. The attitude of the quadrotor is described by the Euler angles $\phi, \theta$ and $\psi$, roll, pitch and yaw, measured respectively over the axes $o_{B} \mathrm{x}_{B}, o_{B} \mathrm{y}_{B}$ and $o_{B} \mathrm{z}_{B}$, with $\left(o_{B} \mathrm{x}_{B} \mathrm{y}_{B} \mathrm{z}_{B}\right)$ being the body axis system whose origin $o_{B}$ is given by the geometric centre of the quadrotor [3]. $u_{\mathrm{x}}, u_{\mathrm{y}}, u_{\mathrm{z}}$ and $u_{\psi}$ are the control actions; in [9], we show the relations between these control actions and the thrusters of the four rotors.

The nonlinear signals, $q_{\mathrm{x}}, q_{\mathrm{y}}, q_{\mathrm{z}}, q_{\theta}, q_{\phi}$, and $q_{\psi}$, are: ${ }^{3}$

$$
\begin{aligned}
\bar{q}_{\mathrm{x}}= & (\theta-\mathrm{c}(\phi) \mathrm{s}(\theta) \mathrm{c}(\psi)-\mathrm{s}(\phi) \mathrm{s}(\psi))+ \\
& (\mathrm{c}(\phi) \mathrm{s}(\theta) \mathrm{c}(\psi)+\mathrm{s}(\phi) \mathrm{s}(\psi))\left(u_{\mathrm{z}} /(M g)+1\right) \\
\bar{q}_{\mathrm{y}}= & (-\phi-\mathrm{c}(\phi) \mathrm{s}(\theta) \mathrm{s}(\psi)+\mathrm{s}(\phi) \mathrm{c}(\psi))+ \\
& (\mathrm{c}(\phi) \mathrm{s}(\theta) \mathrm{s}(\psi)-\mathrm{s}(\phi) \mathrm{c}(\psi))\left(u_{\mathrm{z}} /(M g)+1\right) \\
q_{\mathrm{z}}= & (1-\mathrm{c}(\phi) \mathrm{c}(\theta)) g+(\mathrm{c}(\phi) \mathrm{c}(\theta)-1)\left(u_{\mathrm{z}} / M+g\right) \\
q_{\eta}= & \left(\mathbb{J}^{-1}(\eta)-\mathbb{J}^{-1}(0)\right) \tau-\mathbb{J}^{-1}(\eta) C(\eta, \mathrm{d} \eta / \mathrm{d} t) \mathrm{d} \eta / \mathrm{d} t
\end{aligned}
$$

\footnotetext{
${ }^{1}$ This means that the communication system can operate as either a transmitter or a receiver at any given time, but not simultaneously.

${ }^{2}$ Due to the quadrotor's symmetry, its cross inertia are zero.

${ }^{3}$ The uncertainty signals, $q_{\theta}, q_{\mathrm{x}}, q_{\phi}$ and $q_{\mathrm{y}}$, correspond to the terms which are neglected when linearizing around the equilibrium points $(\theta, \mathrm{x})=(0,0)$ and $(\phi, y)=(0,0)$, and $u_{\mathrm{z}}$ tends to $-M g$.
}

where $\eta=\left[\begin{array}{lll}\phi & \theta & \psi\end{array}\right]^{T}, q_{\eta}=\left[\begin{array}{lll}q_{\phi} & q_{\theta} & q_{\psi}\end{array}\right]^{T}$ and $\mathbb{J}$ and $C(\eta, \mathrm{d} \eta / \mathrm{d} t)$ are the inertial and the Coriolis matrices (see Appendix A of [9] for their definitions).

In this paper, for mathematical simplicity we assume that the nonlinear signals are measured ${ }^{4}$. We propose the following control laws for the $\mathrm{z}$ and $\psi$ dynamics:

$$
\begin{aligned}
u_{\mathrm{z}} & =M\left(\left[\begin{array}{ll}
-a_{\mathbf{z}_{2}} & -a_{\mathrm{z}_{1}}
\end{array}\right] x_{\mathrm{z}}-g-q_{\mathrm{z}}\right), \\
u_{\psi} & =\mathcal{I}_{\mathbf{z}}\left(\left[\begin{array}{ll}
-a_{\psi_{2}} & -a_{\psi_{1}}
\end{array}\right] x_{\psi}-q_{\psi}\right),
\end{aligned}
$$

where $x_{\mathrm{z}}$ and $x_{\psi}$ are the state vectors $\left[\begin{array}{ll}\mathrm{z} & \mathrm{dz} / \mathrm{d} t\end{array}\right]^{T}$ and $\left[\begin{array}{ll}\psi & \mathrm{d} \psi / \mathrm{d} t\end{array}\right]^{T}$, respectively, and $\pi_{\mathrm{z}}(\mathrm{s})=\mathrm{s}^{2}+a_{\mathrm{z}_{1}} \mathrm{~s}+a_{\mathrm{z}_{2}}$ and $\pi_{\psi}(\mathrm{s})=\mathrm{s}^{2}+a_{\psi_{1}} \mathrm{~s}+a_{\psi_{2}}$ are given Hurwitz polynomials. Then, the trajectories $\mathrm{z}$ and $\psi$ satisfy:

$$
\pi_{\mathrm{z}}(\mathrm{d} / \mathrm{d} t) \mathrm{z}=0 \text { and } \pi_{\psi}(\mathrm{d} / \mathrm{d} t) \psi=0 .
$$

Since $\pi_{\mathbf{z}}(\mathrm{s})$ and $\pi_{\psi}(\mathrm{s})$ are Hurwitz, we assume zero initial conditions for $\mathrm{z}$ and $\psi$. Thus, we have that $\mathrm{z}=\mathrm{d} \mathrm{z} / \mathrm{d} t=0$ and $\psi=\mathrm{d} \psi / \mathrm{d} t=0$, and also $u_{\mathrm{z}}=-M g$. We then get the following state space representations:

State Space Representation of the $\mathrm{x}-\theta$ dynamics

$$
\begin{aligned}
& \mathrm{d} x_{\theta} / \mathrm{d} t=A_{\theta} x_{\theta}+B_{\theta}\left(u_{\mathrm{x}}+q_{\theta}\right), \quad \theta=C_{\theta} x_{\theta}, \\
& \mathrm{d} x_{\mathrm{x}} / \mathrm{d} t=A_{\mathrm{x}} x_{\mathrm{x}}+B_{\mathrm{x}}(\mathrm{s}(\theta) \mathrm{c}(\phi)), \quad \mathrm{x}=C_{\mathrm{x}} x_{\mathrm{x}},
\end{aligned}
$$

State Space Representation of the $\mathrm{y}-\phi$ dynamics

$$
\begin{gathered}
\mathrm{d} x_{\phi} / \mathrm{d} t=A_{\phi} x_{\phi}+B_{\phi}\left(u_{\mathrm{y}}+q_{\phi}\right), \quad \phi=C_{\theta} x_{\phi}, \\
\mathrm{d} x_{\mathrm{y}} / \mathrm{d} t=A_{\mathrm{y}} x_{\mathrm{y}}+B_{\mathrm{y}}(\mathrm{s}(\phi)), \quad \mathrm{y}=C_{\mathrm{y}} x_{\mathrm{y}},
\end{gathered}
$$

where $x_{\theta}, x_{\mathrm{x}}, x_{\phi}$ and $x_{\mathrm{y}}$ are the state vectors: $\left[\begin{array}{ll}\theta & \mathrm{d} \theta / \mathrm{d} t\end{array}\right]^{T}, \quad\left[\begin{array}{ll}\mathrm{x} & \mathrm{d} \times / \mathrm{d} t\end{array}\right]^{T}, \quad\left[\begin{array}{ll}\phi & \mathrm{d} \phi / \mathrm{d} t\end{array}\right]^{T}, \quad$ and $\left[\begin{array}{ll}\mathrm{y} & \mathrm{dy} / \mathrm{d} t\end{array}\right]^{T}$, respectively, the maps are defined as follows:

$$
\begin{aligned}
& A_{\theta}=A_{\mathrm{x}}=A_{\phi}=A_{\mathrm{y}}=\left[\begin{array}{ll}
0 & 1 \\
0 & 0
\end{array}\right], B_{\mathrm{y}}=-B_{\mathrm{x}}=g B_{2}, \\
& B_{\theta}=\mathcal{I}_{\mathrm{y}}^{-1} B_{2}, B_{\phi}=\mathcal{I}_{\mathrm{x}}^{-1} B_{2}, B_{2}=\left[\begin{array}{l}
0 \\
1
\end{array}\right], \\
& C_{\theta}=C_{\mathrm{x}}=C_{\phi}=C_{\mathrm{y}}=\left[\begin{array}{ll}
1 & 0
\end{array}\right] .
\end{aligned}
$$

\section{III. $\varepsilon$-RIGHT INVERSE FEEDBACK}

Before stating the main problem addressed in this paper, we present in this section a feedback method to linearize the quadrotor's dynamical model presented in the previous subsection. This linear model will then be used for the formulation of the main problem in the next section.

From (2.4) and (2.6), we note that $q_{\theta}$ and $q_{\phi}$ can be directly compensated with the control actions $u_{\mathrm{x}}$ and $u_{\mathrm{y}}$, respectively. Then, from (2.5) and (2.7), we also note that $q_{\mathrm{x}}$ and $q_{\mathrm{y}}$ could be compensated if we were able to synthesize right-inverses for (2.4) and (2.6). But, the right-inverse of a strictly proper system is a non-proper system, so we have to use a good proper approximation of such a right-inverse.

\footnotetext{
${ }^{4}$ If this assumption does not hold, then the techniques presented in [9] can be used for their compensation.
} 
In the following Theorem (proved in Appendix A), we propose a state feedback which aims to approximately right invert a given strictly proper system.

We assume that:

H1: $\operatorname{ker} B=\{0\}, \operatorname{ker} C^{T}=\{0\}$ and $p \geq m$.

$H 2$ : The pair $(A, B)$ is controllable.

H3: $\Sigma(A, B, C)$ has no system zeros at the origin.

Theorem 1: Let us consider a system described by the state space representation, $\Sigma(A, B, C)$ :

$$
\mathrm{d} x / \mathrm{d} t=A x+B u, \quad y=C x,
$$

where $u \in \mathbb{R}^{m}, x \in \mathbb{R}^{n}$ and $y \in \mathbb{R}^{p}$ are the input, the state and the output, respectively, $A \in \mathbb{R}^{n \times n}, B \in \mathbb{R}^{n \times m}$ and $C$ $\in \mathbb{R}^{p \times n}$. Then, a right inverse of (3.1) is:

$$
\mathbb{N}_{r} \frac{\mathrm{d}}{\mathrm{d} t} x_{r}=\mathbb{A}_{r} x_{r}+\mathbb{B}_{r} \bar{y}, \quad u=\mathbb{C}_{r} x_{r}
$$

where:

$$
\begin{aligned}
& \mathbb{N}_{r}=\left[\begin{array}{cc}
0 & 0 \\
\mathrm{I}_{n} & 0
\end{array}\right], \mathbb{A}_{r}=\left[\begin{array}{cc}
C_{m} & 0 \\
A & B
\end{array}\right], \mathbb{B}_{r}=\left[\begin{array}{c}
-\mathrm{I}_{m} \\
0
\end{array}\right], \\
& \mathbb{C}_{r}=\left[\begin{array}{ll}
0 & \mathrm{I}_{m}
\end{array}\right],
\end{aligned}
$$

and $C_{m}$ is a matrix composed by any $m$ linearly independent rows extracted from $C$.

Moreover, there exist two matrices, $K_{1}(\varepsilon) \in \mathbb{R}^{m \times m}$ and $K_{2}(\varepsilon) \in \mathbb{R}^{n \times m}$, parametrized by a given positive parameter $\varepsilon$, with: $K_{1}(0)=0, K_{2}(0)=0$ and $\operatorname{det}\left(K_{1}(\varepsilon)\right) \neq 0$ for all $\varepsilon>0$, such that the descriptor representation:

$$
\mathbb{N}_{r} \frac{\mathrm{d}}{\mathrm{d} t} \bar{x}_{r}=\overline{\mathbb{A}}_{r}(\varepsilon) \bar{x}_{r}+\mathbb{B}_{r} \bar{y}, \quad u=\mathbb{C}_{r} \bar{x}_{r},
$$

where:

$$
\overline{\mathbb{A}}_{r}(\varepsilon)=\left[\begin{array}{cc}
C_{m} & K_{1}(\varepsilon) \\
A & \left(B+K_{2}(\varepsilon)\right)
\end{array}\right]
$$

is proper, having the characteristic polynomial:

$$
\bar{\pi}(\mathrm{s})=\operatorname{det}\left(\mathrm{s} \mathbb{N}_{r}-\overline{\mathbb{A}}_{r}(\varepsilon)\right)=(\varepsilon \mathrm{s}+1)^{n},
$$

and satisfying

$$
\lim _{\varepsilon \rightarrow 0}\left\|x_{r}(t)-\bar{x}_{r}(t)\right\|=0 .
$$

Furthermore, with the state feedback:

$$
u=\widetilde{K}_{1}^{-1}(\varepsilon) \bar{y}+\left(F_{0}+F_{1}(\varepsilon)\right) x,
$$

where

$$
\operatorname{det}\left(\mathrm{sI}_{n}-\left(A+B F_{0}\right)\right)=\mathrm{s}^{n},
$$

$F_{1}(\varepsilon)=-\widetilde{K}_{1}^{-1}(\varepsilon) C_{m}\left(\mathrm{I}_{n}+\mathcal{C}_{\left(A+B F_{0}, \widetilde{K}_{2}(\varepsilon)\right)} \mathcal{C}_{\left(A+B F_{0}, B\right)}^{r}\right)$,

and $\widetilde{K}_{1}(\varepsilon)$ and $\widetilde{K}_{2}(\varepsilon)$ are the matrices obtained when applying the procedure (3.2) - (3.6) to the state space representation $\Sigma\left(A+B F_{0}, B, C\right)$, we get:

$$
\begin{aligned}
& C\left(\mathrm{~s} \mathrm{I}_{n}-\left(A+B\left(F_{0}+F_{1}(\varepsilon)\right)\right)\right)^{-1} B \widetilde{K}_{1}^{-1}(\varepsilon)= \\
& \left(C\left(\mathrm{~s} \mathrm{I}_{n}-A\right)^{-1} B\right)\left(\mathbb{C}_{r}\left(\mathrm{~s}_{r}-\overline{\mathbb{A}}_{r}(\varepsilon)\right)^{-1} \mathbb{B}_{r}\right) .
\end{aligned}
$$

Let us come back to the state space realizations (2.4) and (2.6). Following the procedure sketched in the proof of Theorem 1, we get from $(2.8):^{5}$

$$
\begin{aligned}
& \widetilde{K}_{1}(\varepsilon)=\left(\ell / I_{o}\right) \varepsilon^{2}, \widetilde{K}_{2}(\varepsilon)=\left[\begin{array}{c}
2\left(\ell / I_{o}\right) \varepsilon \\
0
\end{array}\right], \\
& \mathcal{C}_{\left(A, \widetilde{K}_{2}(\varepsilon)\right)}=\left[\begin{array}{cc}
2\left(\frac{\ell}{I_{o}}\right) \varepsilon & 0 \\
0 & 0
\end{array}\right], \mathcal{C}_{(A, B)}^{r}=\left(\frac{I_{o}}{\ell}\right)\left[\begin{array}{ll}
0 & 1 \\
1 & 0
\end{array}\right],
\end{aligned}
$$

where $\varepsilon>0$. From (3.10) and (3.12), we get the following control laws for the $\mathrm{x}-\theta$ and $\mathrm{y}-\phi$ dynamics:

$$
\begin{aligned}
& u_{\mathrm{x}}=\mathcal{I}_{\mathrm{y}}\left(-q_{\theta}+\left[\begin{array}{ll}
-1 / \varepsilon^{2} & -2 / \varepsilon
\end{array}\right] x_{\theta}-\frac{1}{\varepsilon^{2}} \mathrm{~s}^{-1}\left(\frac{\bar{\theta}}{\mathrm{c}(\phi)}\right)\right), \\
& u_{\mathrm{y}}=\mathcal{I}_{\mathrm{x}}\left(-q_{\phi}+\left[\begin{array}{ll}
-1 / \varepsilon^{2} & \left.-2 / \varepsilon] x_{\phi}+\frac{1}{\varepsilon^{2}} \mathrm{~s}^{-1}(\bar{\phi})\right)
\end{array},\right.\right.
\end{aligned}
$$

where $\varepsilon$ is a positive parameter depending on the bandwidth of the system represented by (2.4)-(2.7), and $\bar{\theta}$ and $\bar{\phi}$ are the new control signals. Applying the control laws (3.13) to (2.4)-(2.5) and (2.6)-(2.7), respectively, we get:

$$
\begin{aligned}
\mathrm{d}^{2} \mathrm{x} / \mathrm{d} t^{2} & =g(\mathrm{~s}(\theta) \mathrm{c}(\phi)), \\
(\varepsilon \mathrm{d} / \mathrm{d} t+1)^{2} \theta & =\mathrm{s}^{-1}(\bar{\theta} / \mathrm{c}(\phi)), \\
\mathrm{d}^{2} \mathrm{y} / \mathrm{d} t^{2} & =g(\mathrm{~s}(\phi)), \\
(\varepsilon \mathrm{d} / \mathrm{d} t+1)^{2} \phi & =\mathrm{s}^{-1}(\bar{\phi}) .
\end{aligned}
$$

The control signals $\mathrm{s}^{-1}(\bar{\theta} / \mathrm{c}(\phi))$ and $\mathrm{s}^{-1}(\bar{\phi})$ are related to $\theta_{\mathrm{x}}$ and $\phi_{\mathrm{y}}$ by means of the low-pass filter $1 /(\varepsilon \mathrm{s}+1)^{2}$, with the cut-off frequency: $\omega_{c}=1 / \varepsilon$. Thus, if the control signals, $\mathrm{s}^{-1}(\bar{\theta} / \mathrm{c}(\phi))$ and $\mathrm{s}^{-1}(\bar{\phi})$, are bounded and band limited signals, with a bandwidth $\omega_{q}$, then the cut-off frequency $\omega_{c}$ should be set sufficiently greater than $\omega_{q}$. In this case, (3.14) can be expressed as follows:

$$
\left(\mathrm{d}^{2} \mathrm{x} / \mathrm{d} t^{2}-g \bar{\theta}\right)=\mathcal{O}(\varepsilon), \quad\left(\mathrm{d}^{2} \mathrm{y} / \mathrm{d} t^{2}-g \bar{\phi}\right)=\mathcal{O}(\varepsilon) .
$$

Finally, in order to ensure the proper operation of the quadrotor, we limit the range of variations of the pitch and roll angles. Since both angles are decoupled in the linearized model (3.15), we constrain them separately as follows $\|\bar{\phi}\|_{\infty} \leq \Phi$ and $\|\bar{\theta}\|_{\infty} \leq \Theta$. Now that we have a linear model for the quadrotor's position in the $x-y$ plane (i.e., $\mathbf{p}(t)$ ), we proceed to state, in the next section, the main problem addressed in this paper.

\section{PROBLEM STATEMENT}

We now present the main problem addressed in this paper which consists of the optimization of the quadrotor's periodic trajectory so that it maximizes the amount of information transferred from the SN to the DN per period. Each period, of duration $T$, is divided into two phases. During the first phase,

$$
\begin{aligned}
& \quad{ }^{5} \text { Do: } n=2, m=1, A_{\theta} \mapsto A, B_{\theta} \mapsto B, C_{\theta} \mapsto C_{m}, T_{L}=\mathrm{I}_{3}, T_{R} \\
& =\left[\begin{array}{ccc}
1 & 0 & 0 \\
0 & 1 & 0 \\
0 & 0 & I_{o} / \ell
\end{array}\right], P=\left[\begin{array}{lll}
0 & 0 & 1
\end{array}\right], \bar{K}_{1}(\varepsilon)=\varepsilon^{2}, \text { and } \bar{K}_{2}(\varepsilon)= \\
& {\left[\begin{array}{c}
2 \varepsilon \\
0
\end{array}\right] \text {. Note that: } F_{0}=0 .}
\end{aligned}
$$




\section{CONFIDENTIAL. Limited circulation. For review only.}

the quadrotor receives data from the $\mathrm{SN}$, and during the second phase, it transfers all this data to the DN. To quantify the achievable bit rate, we use Shannon channel capacity formula for a time-invariant AWGN channel ${ }^{6}$ [13]. Hence the bit rates of the communications through the channels SN-to-quadrotor $(k=0)$ and quadrotor-to-DN $(k=1)$ are:

$$
R\left(\mathbf{p}(t), \mathbf{p}_{k}\right)=B \log _{2}\left(1+\Gamma\left(\mathbf{p}(t), \mathbf{p}_{k}\right)\right), \quad k=0,1
$$

where $B$ is the bandwidth of the signals used in the data transmission. Hence, the upper bound of the number of bits that can be transmitted during one period (with an arbitrarily small error) from the $\mathrm{SN}$ to the quadrotor and from the quadrotor to the DN are $\int_{0}^{T}(1-S W(t)) R\left(\mathbf{p}(t), \mathbf{p}_{0}\right) \mathrm{d} t$ and $\int_{0}^{T} S W(t) R\left(\mathbf{p}(t), \mathbf{p}_{1}\right) \mathrm{d} t$ respectively. Also, we remind the reader that the quadrotor has to transmit to the $\mathrm{DN}$ all the data received from the $\mathrm{SN}$.

To simplify the problem, we assume that:

H4: The quadrotor cannot get closer than a distance $d_{\text {min }}$ from the $\mathrm{SN}$ and from the $\mathrm{DN}$ due to physical limitations (e.g., fences, trees, cables, ...). We will refer to the circles marking those limits as SN and DN borders.

So, the trajectory optimization problem mentioned above can be formally stated as follows.

Problem 1: Optimize the quadrotor's periodic trajectory in order to maximize the upper bound on the number of bits than can be transferred by the quadrotor from the $\mathrm{SN}$ to the DN.

$$
\max _{\bar{\theta}, \bar{\phi}, \beta, y_{0}, v_{0}^{\times}, v_{0}^{y}} \int_{0}^{T}(1-S W(t)) R\left(\mathbf{p}(t), \mathbf{p}_{0}\right) \mathrm{d} t
$$

subject to the following constraints

$$
\begin{aligned}
& \int_{0}^{T}(1-S W(t)) R\left(\mathbf{p}(t), \mathbf{p}_{0}\right) \mathrm{d} t=\int_{0}^{T} S W(t) R\left(\mathbf{p}(t), \mathbf{p}_{1}\right) \mathrm{d} t \\
& \left\|\mathbf{p}(t)-\mathbf{p}_{0}\right\|_{2} \geq d_{m i n},\left\|\mathbf{p}(t)-\mathbf{p}_{1}\right\|_{2} \geq d_{m i n} \forall t \in[0, T] \\
& \mathrm{d}^{2} \mathrm{x}(t) / \mathrm{d} t^{2}=g \bar{\theta}, \mathrm{d}^{2} \mathrm{y}(t) / \mathrm{d} t^{2}=g \bar{\phi}, \\
& \mathrm{x}(0)=\mathrm{x}(T)=x_{0}, \mathrm{y}(0)=\mathrm{y}(T)=\mathrm{y}_{0}, \\
& \mathrm{~d} \times(0) / \mathrm{d} t=\mathrm{d} \times(T) / \mathrm{d} t=v_{0}^{\mathrm{x}}, \mathrm{dy}(0) / \mathrm{d} t=\operatorname{dy}(T) / \mathrm{d} t=v_{0}^{\mathrm{y}}, \\
& \|\bar{\theta}\|_{\infty} \leq \Theta, \quad\|\bar{\phi}\|_{\infty} \leq \Phi, \\
& S W(t)=0, \forall t \in[0, \beta T), S W(t)=1, \forall t \in[\beta T, T),
\end{aligned}
$$

where $\beta \in(0,1), x_{0}, \mathrm{y}_{0}, v_{0}^{\mathrm{x}}$ and $v_{0}^{\mathrm{y}}$ are parameters describing the initial state of the quadrotor.

Remark 1: (i) The first line of constraints ensures that all the data collected by the quadrotor from the $\mathrm{SN}$ is transferred to the DN. (ii) The second line of constraints ensures that the quadrotor maintains a minimum distance of $d_{\text {min }}$ from both the SN and DN. (iii) The third line of constraints describe the resulting dynamical model of the quadrotor after the

\footnotetext{
${ }^{6}$ The channel capacity determines the maximum bit rate achievable with an arbitrarily small error rate when communicating through the considered channel. A deeper discussion on the use of this formula in our communication scenario and its implications is outside the scope of this paper.
}

linearization through the feedback introduced in section III. (iv) The fourth and fifth lines are required for the periodicity of the trajectory. $(v)$ The next line of constraints limits the maximum variations of the pitch and roll angles. (vi) The final set of constraints describes the intervals of time in which the quadrotor's communications system operates as a receiver and then as a transmitter.

During $t \in[0, \beta T)(t \in[\beta T, T))$ the quadrotor communicates with the $\mathrm{SN}$ (DN) with an instantaneous bit rate $R\left(\mathbf{p}(t), \mathbf{p}_{0}\right)\left(R\left(\mathbf{p}(t), \mathbf{p}_{1}\right)\right)$. Now, from the definitions of $\mathbf{p}_{0}$ and $\mathbf{p}_{1}$ in section II-A we note that

$$
R\left(\mathbf{p}(t), \mathbf{p}_{0}\right)>R\left(\mathbf{p}(t), \mathbf{p}_{1}\right) \text { for } x(t)>0
$$

and

$$
R\left(\mathbf{p}(t), \mathbf{p}_{1}\right)>R\left(\mathbf{p}(t), \mathbf{p}_{0}\right) \text { for } x(t)<0 .
$$

To solve (4.2), (4.3), it is clear that the optimum trajectory must maximize $R\left(\mathbf{p}(t), \mathbf{p}_{0}\right)\left(R\left(\mathbf{p}(t), \mathbf{p}_{1}\right)\right.$ during $t \in[0, \beta T)$ $(t \in[\beta T, T))$. This implies that the optimum quadrotor's trajectory satisfies:

$$
x(t)>0 \text { for } t \in(0, \beta T) \text { and } x(t)<0 \text { for } t \in(\beta T, T) .
$$

Then, considering the periodicity of the trajectory, we have that:

$$
\begin{gathered}
\mathrm{x}(0)=\mathrm{x}(\beta T)=\mathrm{x}(T)=0, \\
\mathrm{~d} \times(0) / \mathrm{d} t=\mathrm{d} \times(T) / \mathrm{d} t \geq 0, \mathrm{~d} \times(\beta T) / \mathrm{d} t \leq 0, \\
\mathrm{x}(t) \geq 0, \forall t \in[0, \beta T), \mathrm{x}(t) \leq 0, \forall t \in[\beta T, T) .
\end{gathered}
$$

In the following Theorem (proved in Appendix B), we state some optimum trajectory properties which enable us to further simplify Problem 1 before attempting to solve it.

Theorem 2 (Optimum trajectory properties): If $\mathrm{y}_{0}=-\mathrm{y}_{1}, v_{0}^{\mathrm{x}}=-v_{1}^{\mathrm{x}}$ and $v_{0}^{\mathrm{y}}=-v_{1}^{\mathrm{y}}$, both phases of the optimal trajectory must have the same duration, i.e. $\beta=0.5$. Hence, both phases of the optimum trajectory $\left[\mathrm{x}^{*} \mathrm{y}^{*}\right]^{\mathrm{T}}$ are, $\forall t \in[0, T / 2)$, are related according to

$$
\mathrm{x}^{*}(t+T / 2)=-\mathrm{x}^{*}(t), \quad \mathrm{y}^{*}(t+T / 2)=-\mathrm{y}^{*}(t) .
$$

Corollary 1: Under the conditions stated in Theorem 2, the optimization problem (4.2)-(4.3) is simplified to:

$$
\max _{\bar{\theta}, \bar{\phi}} \int_{0}^{T / 2} \log _{2}\left(1+\frac{P / \sigma^{2}}{(D / 2-\mathrm{x}(t))^{2}+\mathrm{y}^{2}(t)}\right) \mathrm{d} t
$$

subject to the following constraints

$$
\begin{aligned}
& (D / 2-\mathrm{x}(t))^{2}+\mathrm{y}^{2}(t) \geq d_{\text {min }}^{2} \forall t \in[0, T / 2], \\
& \mathrm{d}^{2} \mathrm{x}(t) / \mathrm{d} t^{2}=g \bar{\theta}, \mathrm{d}^{2} \mathrm{y}(t) / \mathrm{d} t^{2}=g \bar{\phi}, \\
& \mathrm{x}(0)=\mathrm{x}(T / 2)=0, \mathrm{y}(0)=-\mathrm{y}(T / 2), \\
& \mathrm{d} \times(0) / \mathrm{d} t=-\mathrm{d} \times(T / 2) / \mathrm{d} t, \quad \mathrm{dy}(0) / \mathrm{d} t=-\mathrm{dy}(T / 2) / \mathrm{d} t \\
& \|\bar{\theta}\|_{\infty} \leq \Theta, \quad\|\bar{\phi}\|_{\infty} \leq \Phi,
\end{aligned}
$$

Proof: By setting $\mathrm{y}_{0}=-\mathrm{y}_{1}, v_{0}^{\mathrm{x}}=-v_{1}^{\mathrm{x}}$ and $v_{0}^{\mathrm{y}}=-v_{1}^{\mathrm{y}}$ according to Theorem 2, we have that $\beta^{*}=0.5$ in (4.2)-(4.3), 


\section{CONFIDENTIAL. Limited circulation. For review only.}

i.e., both phases of the trajectory have the same duration. In addition, both trajectory phases are related according to (4.5). This relation automatically satisfies the constraint that the quadrotor must transmit to the DN the same number of bits received from the SN. Hence, by taking all this into consideration, the optimization problem (4.2)-(4.3) becomes (4.6)-(4.7) after setting $\mathrm{y}_{0}=-\mathrm{y}_{1}, v_{0}^{\mathrm{x}}=-v_{1}^{\mathrm{x}}$ and $v_{0}^{\mathrm{y}}=-v_{1}^{\mathrm{y}}$.

\section{PROBLEM SOLUTION}

The optimization problem (4.6)-(4.7) could be solved numerically using dynamic programming [14]. However, such an approach is computationally too expensive. We derive a suboptimal (but with much lower computational load than dynamic programming) solution by approximating $x$ and $y$ with a Fourier series of order $N$. The resulting optimization problem is:

$$
\max _{\left\{a_{k}, b_{k}, c_{k}, d_{k}\right\}_{k=1}^{N}} \int_{0}^{T / 2} \log _{2}\left(1+\frac{P / \sigma_{n}^{2}}{(D / 2-\overline{\mathrm{x}}(t))^{2}+\overline{\mathrm{y}}^{2}(t)}\right) \mathrm{d} t
$$

subject to the following constraints

$$
\begin{aligned}
& \overline{\mathrm{x}}(t)=\sum_{k=1}^{N}\left(a_{k} \cos (k \omega t)+b_{k} \sin (k \omega t)\right), \\
& \overline{\mathrm{y}}(t)=\sum_{k=1}^{N}\left(c_{k} \cos (k \omega t)+d_{k} \sin (k \omega t)\right), \\
& \|\overline{\mathrm{x}}(t)\|_{\infty} \leq g \Theta,\|\overline{\mathrm{y}}(t)\|_{\infty} \leq g \Phi, \\
& (D / 2-\overline{\mathrm{x}}(t))^{2}+\overline{\mathrm{y}}^{2}(t) \geq d_{\text {min }}^{2} \forall t \in[0, T / 2], \\
& \sum_{k \in \mathrm{E}_{N}} a_{k}=0, \quad \sum_{k \in \mathrm{O}_{N}} a_{k}=0, \quad \sum_{k \in \mathrm{E}_{N}} c_{k}=0, \\
& \sum_{k \in \mathrm{E}_{N}} k b_{k}=0, \quad \sum_{k \in \mathrm{E}_{N}} k d_{k}=0,
\end{aligned}
$$

where $O_{N}$ and $E_{N}$ represent the set of all odd and even numbers in the set $\{1,2, \cdots, N\}$. The first two lines of constraints describe the parametric representation of the trajectory using Fourier series of order $N$ where $\omega=2 \pi / T$. The next line of constraints ensures that the quadrotor will maintain a minimum distance of $d_{\min }$ from the $\mathrm{SN}$ and the DN. Then, the next set of constraints on the Fourier series coefficients ensure that the initial and final conditions on $\bar{x}(t)$ and $\bar{y}(t)$ (as well as on their derivative) are met $^{7}$.

It is worth pointing out that one of the main advantages of approximating the trajectory using the Fourier series is that the resulting trajectory will be infinitely derivable and thus it can be tracked by the quadrotor without any problem, see (3.15).

\section{A. Suboptimal Trajectory}

In order to solve problem (5.1), (5.2), whose solution is an approximation of the solution of (4.6), (4.7), we used simulated annealing (SA) algorithm [15]. This optimization algorithm combines a random and gradient-like search procedures in order to find the optimum value.

We solve (5.1), (5.2) for: $P / \sigma^{2}=10^{3}, D / 2=400[\mathrm{~m}]$, $T=300[\mathrm{~s}], d_{\min }=30[\mathrm{~m}]$ and $\Theta=\Phi=\pi / 18$. After

\footnotetext{
${ }^{7}$ To differentiate the actual quadrotor's position $\mathbf{p}(t)=[\mathrm{xy}]^{\mathrm{T}}$ from the trajectory designed by solving (5.1), (5.2) we will denote the latter by $\overline{\mathbf{p}}(t)=[\bar{x} \bar{y}]^{\mathrm{T}}$.
}

running multiple times the SA to optimize (5.1), (5.2), we observed the presence of various local maxima. We present in Table I the value $\mathcal{J}$ of the optimization $\operatorname{target}^{8}$ (i.e., the upper bound on the number of bits transmitted during the trajectory, normalized by the transmission bandwidth $B$ ) resulting from the optimum trajectory for different values of $N^{9}$. For comparison purposes, we also present $\mathcal{R}$ in Table I which corresponds to the ratio of $\mathcal{J}$ over the upper bound on the number of bits that the quadrotor would be able to transfer if it remained still at the origin (i.e., if $\overline{\mathbf{p}}(t)=\left[\begin{array}{ll}0 & 0\end{array}\right]^{\mathrm{T}}$ for all $t$ ). By observing $\mathcal{R}$ in Table I, we note that the set of considered suboptimal trajectories produce a large improvement in the amount of data transmitted with respect to the case in which the quadrotor remains still at the origin. In Fig. 1 we observe, for different values of $N$, the normalized channel capacity that the quadrotor would see during the first phase of the optimum trajectory.

We note from Fig. 1 that the channel capacity profile seen by the quadrotor during the first phase ${ }^{10}$ is divided in three parts. In the first part the quadrotor accelerates to get as close as possible from the SN (hence the fast initial increase of the channel capacity). In the second part, the quadrotor tries to stay as long as possible close to the $\mathrm{SN}$ (hence a duration of high channel gain). Then in the third part the quadrotor moves away quickly from the $\mathrm{SN}$ (towards the $\mathrm{DN}$ ); by doing this, the quadrotor can stay longer close to the $\mathrm{SN}$ and hence maximize the number of bits received during the first phase of the trajectory.

Note, from Fig. 1, that as $N$ increases the rise on the channel capacity tends to become sharper and to happen earlier. Note also that as $N$ increases, the initial rise on the channel capacity profile will reach a limit once the acceleration of the quadrotor reaches its limit. Also, as $N$ increases, the center of the channel capacity profile tends to become flatter. All these elements help us to infer the shape of the channel capacity profile resulting from the optimum trajectory obtained by solving (4.2)-(4.3).

In Fig. 1, we also present the path (for $t \in[0, T / 2]$ ) described by the quadrotor's optimum trajectory for $N=6$. The reason why the quadrotor moves on a straight line tangential to the SN border rather than on a straight line orthogonal to the SN border is the fact that the constraints on the pitch and roll angles are independent (i.e., $\|\bar{\phi}\|_{\infty}<\Phi$ and $\left.\|\bar{\theta}\|_{\infty}<\Theta\right)$.

Finally, we have to mention that the delay introduced by this method into the transmission of the data from the SN to the DN via the quadrotor is $T / 2=150$ s. Note that such delays are acceptable in for delay-tolerant applications.

\footnotetext{
${ }^{8}$ We present the largest maximum that we have found after running the SA algorithm numerous times.

${ }^{9}$ Due to the initial and final state constraints on the trajectory, the values of the Fourier series coefficients are the same for $N=1$ and $N=2$.

${ }^{10}$ The trajectory of the transmission phase is not shown since it is symmetric to the trajectory during the reception phase according to Theorem
} 2 . 
TABLE I

Maxima $\mathcal{J}$ AND $\mathcal{R}$ vs $N$

\begin{tabular}{||c||c|c|c|c|c|c||}
\hline \hline$N$ & 1 & 3 & 4 & 5 & 6 & 7 \\
\hline $\mathcal{J}$ & 54.96 & 95.43 & 101.98 & 111.72 & 115.38 & 120.71 \\
\hline $\mathcal{R}$ & 40.75 & 70.78 & 76.60 & 82.88 & 85.46 & 89.32 \\
\hline \hline
\end{tabular}

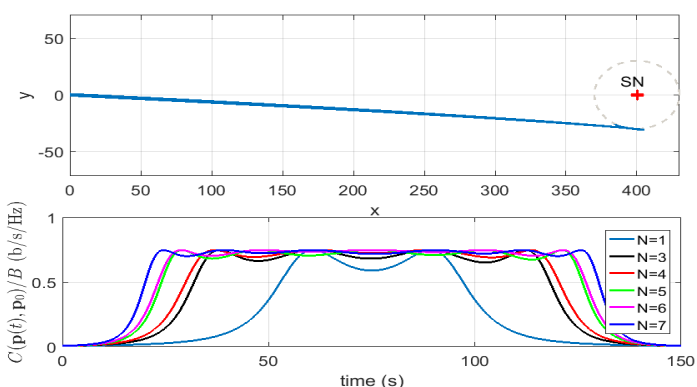

Fig. 1. Top: path described by optimum $\overline{\mathbf{p}}(t)$ for $N=6$ and $t \in[0, T], \mathrm{SN}$ and $\mathrm{DN}$ are marked in red and minimum distance between these nodes and the quadrotor is marked by gray circles. Bottom: instantaneous bit rate achievable for different values of $N$ and $t \in[0, T / 2]$.

\section{B. Suboptimal Trajectory Tracking}

In order for the quadrotor to track the suboptimal trajectory $\overline{\mathbf{p}}(t)$ we use a control scheme composed by an internal static state feedback (ISSF), coming from $x_{\theta}$ and $x_{\phi}$, and an external dynamic state feedback (EDSF), coming from $x_{\mathrm{x}}$ and $x_{\mathrm{y}}$.

a) Internal static state feedbacks: The ISSF are composed by (3.13). We set: $\varepsilon=1 / 100$.

b) External Dynamic state feedbacks: The EDSF are composed by the cascade of a state feedback with a lead network:

$$
(\mathrm{d} / \mathrm{d} t+1 /(10 \varepsilon))\left(v_{i}-\bar{u}_{i}\right)=-(\mathrm{d} / \mathrm{d} t+b)\left[\begin{array}{ll}
f_{1} & f_{2}
\end{array}\right] x_{i},
$$

where: $i \in\{\mathrm{x}, \mathrm{y}\}, v_{\mathrm{x}}=\bar{\theta}$ and $v_{\mathrm{y}}=\bar{\phi}$.

From (5.3) and (3.15), we get the following transfer functions $(i \in\{\mathrm{x}, \mathrm{y}\})$ :

$$
\begin{aligned}
& T_{i}(\mathrm{~s})= \\
& \frac{g(10 \varepsilon \mathrm{s}+1)}{\left(\varepsilon^{2} \mathrm{~s}+1\right)^{2}(10 \varepsilon \mathrm{s}+1) \mathrm{s}^{2}+g\left(f_{1}+f_{2} \mathrm{~s}\right)(\mathrm{s}+b)} \\
& \approx \frac{g}{\left(1+k_{c}\right) \mathrm{s}^{2}+k_{c}\left(b+f_{1} / f_{2}\right) \mathrm{s}+k_{c} b f_{1} / f_{2}} \\
& \approx \frac{g /\left(1+k_{c}\right)}{(\mathrm{s}+\bar{a})^{2}}, \quad k_{c}=10 \varepsilon f_{2} g .
\end{aligned}
$$

Following a root locus design, we have chosen $f_{1}=0.5944$, $f_{2}=1.1888$ and $b=2.4423$, . For the closed loop poles location: $\{-130.73,-38.78,-38.78,-0.85,-0.85\}$. We set: $\bar{a}=0.85$.

Thus, doing: ${ }^{11}$

$$
\begin{aligned}
& \bar{u}_{\mathrm{x}}=\left(\left(1+k_{c}\right) / g\right)\left(\mathrm{d}^{2} \overline{\mathrm{x}} / \mathrm{d} t^{2}+2 \bar{a} \mathrm{~d} \overline{\mathrm{x}} / \mathrm{d} t+\bar{a}^{2} \overline{\mathrm{x}}\right), \\
& \bar{u}_{\mathrm{y}}=\left(\left(1+k_{c}\right) / g\right)\left(\mathrm{d}^{2} \overline{\mathrm{y}} / \mathrm{d} t^{2}+2 \bar{a} \mathrm{~d} \overline{\mathrm{y}} / \mathrm{d} t+\bar{a}^{2} \overline{\mathrm{y}}\right),
\end{aligned}
$$

\footnotetext{
${ }^{11}$ Since $\bar{x}$ and $\bar{y}$ are expressed by Fourier series, then their time derivatives are easily computed.
}
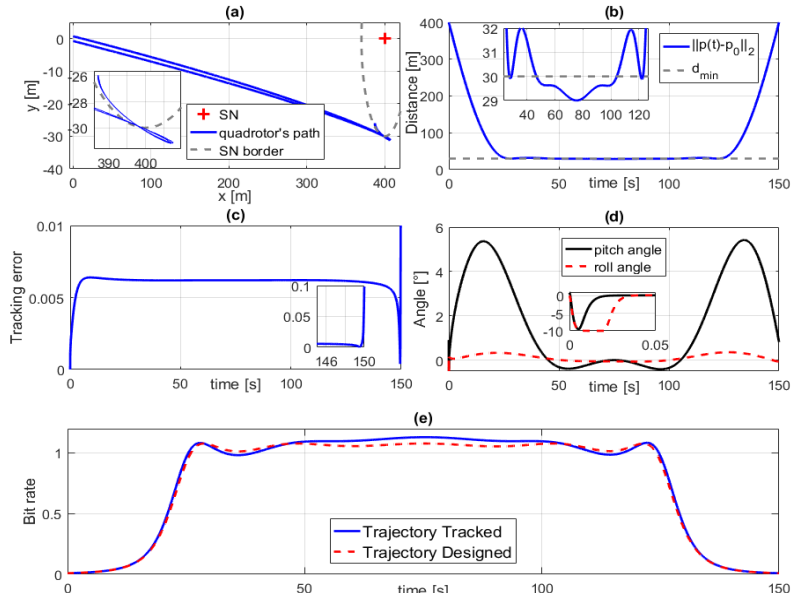

Fig. 2. Quadrotor's behaviour during the tracking of $\overline{\mathbf{p}}(t)$ for $t \in[0, T / 2]$ : (a) y vs. $\times[\mathrm{m}]$. (b) $\left\|\mathbf{p}(t)-\mathbf{p}_{0}\right\|_{2}[\mathrm{~m}]$. (c) $\|\mathbf{p}(t)-\overline{\mathbf{p}}(t)\|_{2} /\|\overline{\mathbf{p}}(t)\|_{2}$ [m]. (d) pitch $\theta$ and roll $\phi$ angles $\left[^{\circ}\right]$. (e) $R\left(\mathbf{p}(t), \mathbf{p}_{0}\right) / B$ in blue; $R\left(\overline{\mathbf{p}}(t), \mathbf{p}_{0}\right) / B$ in red

we finally get:

$$
\mathrm{x}=\overline{\mathrm{x}}+k_{\mathrm{x}} \mathrm{e}^{-\bar{a} t}+\mathcal{O}(\varepsilon), \quad \mathrm{x}=\overline{\mathrm{x}}+k_{\mathrm{y}} \mathrm{e}^{-\bar{a} t}+\mathcal{O}(\varepsilon),
$$

where $k_{\mathrm{x}}$ and $k_{\mathrm{y}}$ are constants depending on the initial conditions.

Finally, we compare how well the quadrotor can track ${ }^{12}$ $\overline{\mathbf{p}}(t)$ for $N=6$. To do this, we consider a quadrotor model with parameter values extracted from a laboratory prototype (see [9]): $M=1.36[\mathrm{~kg}], g=9.81\left[\mathrm{~m} \mathrm{~s}^{-2}\right], I_{\times}=$ $0.0134\left[\mathrm{~kg} \mathrm{~m}^{2}\right], I_{\mathrm{y}}=0.0140\left[\mathrm{~kg} \mathrm{~m}^{2}\right], I_{\mathrm{z}}=0.0256\left[\mathrm{~kg} \mathrm{~m}^{2}\right], L$ $=0.0245[\mathrm{~m}]$.

From Figs. 2, we observe that the quadrotor has no problem in following the trajectory designed $(\overline{\mathbf{p}}(t))$ (tracking error below 1\%). The constraints regarding the maximum roll and pitch angle variations are met and the quadrotor gets closer to the SN than allowed only by a small distance (see Fig. 2 (b)). In Fig. 2 (e), we observe the instantaneous bit rate profile experienced by the quadrotor during the trajectory tracking which is significantly similar to the one associated to $\overline{\mathbf{p}}(t)$. Finally, the optimization target evaluated on $\overline{\mathbf{p}}(t)$ is $\overline{\mathcal{J}}=115.38$ bits per $\mathrm{Hz}$ and on $\mathbf{p}(t)$ is $\mathcal{J}=117.5$ bits per Hz. Therefore, we can say that from a communications perspective, the performance of the trajectory described by the quadrotor $(\mathbf{p}(t))$ is close enough to the performance of the trajectory designed $\overline{\mathbf{p}}(t)$.

\section{CONCLUSION}

We successfully optimized a periodic trajectory for a quadrotor so that it maximizes the amount of data transferred

\footnotetext{
${ }^{12}$ The Fourier coefficients, for $N=6$, obtained by executing the SA algorithm to solve (5.1), (5.2) are:

$\left(a_{1}, b_{1}, c_{1}, d_{1}\right)=(-2.0907,478.1504,0.1765,-35.3231)$, $\left(a_{2}, b_{2}, c_{2}, d_{2}\right)=(-10.4373,3.0189,-1.7834,-0.6714)$ $\left(a_{3}, b_{3}, c_{3}, d_{3}\right)=(2.0285,136.9332,-0.6628,-7.8130)$, $\left(a_{4}, b_{4}, c_{4}, d_{4}\right)=(-0.0737,-1.1088,1.7767,0.2081)$ $\left(a_{5}, b_{5}, c_{5}, d_{5}\right)=(0.0621,43.6466,-0.2366,-1.8607)$, $\left(a_{6}, b_{6}, c_{6}, d_{6}\right)=(10.5110,-0.2671,0.0066,0.0851)$
} 


\section{CONFIDENTIAL. Limited circulation. For review only.}

from a source node to a destination in a delay tolerant application. The optimized trajectory results in a significant increment on the amount of data transferred and is easily trackable by the quadrotor. The trajectory is parametrized with a Fourier series whose parameters are optimized. This parametric approach results also in a low computational load. Future work will address the issue of multiple maxima within the optimization problem, the energy consumption of the quadrotor and experimental validation.

\section{APPENDIX}

\section{A. Proof of Theorem 1}

a) Right Inverse: In the Fact 1 of [5], we proved that (3.2) is a one side inverse of (3.1) (see also Lemma 3 of [8]).

b) Proper Approximation: Let us note that $\mathbb{N}_{r}$ is a nilpotent matrix and that $H 3$ implies the invertibility of $\mathbb{A}_{r}$.

There then exist changing bases matrices, $T_{L}$ and $T_{R}$, such that the regular pencil $\left[\mathrm{s} \mathbb{N}_{r}-\mathbb{A}_{r}(\varepsilon)\right]$ is transformed in its Weierstrass form [10]: $T_{L}\left[\mathrm{~s} \mathbb{N}_{r}-\mathbb{A}_{r}\right] T_{R}=\left[\mathrm{s} \mathbb{N}_{r}-\mathrm{I}\right]$.

Following the procedure proposed in Theorem 2.1 of [6], we get the proper pencil $\left[\mathrm{s} \mathbb{N}_{r}-J(\varepsilon)\right]$, where: $J(\varepsilon)=\mathrm{I}+$ $\bar{K}(\varepsilon) P, P \in \mathbb{R}^{m \times n}$ is the natural projection on ker $\mathbb{N}_{r}$, and the matrix, $\bar{K}(\varepsilon)=\left[\begin{array}{l}\bar{K}_{1}(\varepsilon) \\ \bar{K}_{2}(\varepsilon)\end{array}\right], \bar{K}_{1}(\varepsilon) \in \mathbb{R}^{m \times m}, \bar{K}_{2}(\varepsilon)$ $\in \mathbb{R}^{(n-m) \times m}$, is a matrix, parametrized by a given positive parameter $\varepsilon$, such that: $\operatorname{det}\left(\mathrm{s} \mathbb{N}_{r}-J(\varepsilon)\right)=(\varepsilon \mathrm{s}+1)^{n}, \bar{K}_{1}(\varepsilon)$ $=\varepsilon \mathrm{I}_{m}$ and $\bar{K}(0)=0$.

Coming back to the initial bases (in the domain and the co-domain), we get (3.4) - (3.6), together with: $K_{1}(0)=0$ and $K_{2}(0)=0$. (3.7) is also proved in Theorem 2.1 of [6].

On the other hand, since (3.4) is an internally proper ${ }^{13}$ description for all $\varepsilon>0$, we have from Proposition 9 of [7], that: $\operatorname{ker}\left[\begin{array}{ll}\mathrm{I}_{n} & 0\end{array}\right] \oplus \operatorname{ker}\left[\begin{array}{ll}C_{m} & K_{1}(\varepsilon)\end{array}\right]=\mathbb{R}^{(n+m) \text {, }}$ namely: $\operatorname{ker}\left[\begin{array}{cc}\mathrm{I}_{n} & 0\end{array}\right] \cap \operatorname{ker}\left[\begin{array}{cc}C_{m} & K_{1}(\varepsilon)\end{array}\right]=\{0\}$, which implies: $\operatorname{ker} K_{1}(\varepsilon)=\{0\}$ for all $\varepsilon>0$.

c) $\varepsilon$-Right Inverse Feedback: H2 implies the existence of $F_{0}$ satisfying (3.9), and the existence of a right inverse $\mathcal{C}_{\left(A_{0}, B\right)}^{r}$, where $A_{0}=A+B F_{0}$, namely: $\mathcal{C}_{\left(A_{0}, B\right)} \mathcal{C}_{\left(A_{0}, B\right)}^{r}=$ $\mathrm{I}_{n}$. Defining the first state feedback: $u=u_{0}+F_{0} x$, we get:

$$
\mathrm{d} x / \mathrm{d} t=A_{0} x+B u_{0} .
$$

From the inversion Lemma and recalling the CayleyHamilton Theorem, we deduce (see for example [11], [12]):

$$
\begin{aligned}
& x(\mathrm{~s})=\left(\mathrm{s} \mathrm{I}_{n}-A_{0}\right)^{-1} B u_{0}(\mathrm{~s})=\sum_{i=1}^{n} A_{0}^{i-1} B \mathrm{~s}^{-i} u_{0}(\mathrm{~s}) \\
& x(\mathrm{~s})=\mathcal{C}_{\left(A_{0}, B\right)} \Psi_{m}\left(\mathrm{~s}^{-1}\right) u_{0}(\mathrm{~s}) \\
& \Psi_{m}\left(\mathrm{~s}^{-1}\right) u_{0}(\mathrm{~s})=\mathcal{C}_{\left(A_{0}, B\right)}^{r} x(\mathrm{~s}) .
\end{aligned}
$$

On the other hand, applying the procedure (3.2) - (3.6) to $\Sigma\left(A_{0}, B, C\right)$, we get, after algebraic reductions, the follow-

\footnotetext{
${ }^{13}$ The pencil $\left[\mathrm{s} \mathbb{N}_{r}-\overline{\mathbb{A}}_{r}(\varepsilon)\right]$ is regular and it has no infinite zeros of order greater than one (there are no derivators) [2], [1].
}

ing right inverse approximation:

$$
\begin{aligned}
\frac{\mathrm{d}}{\mathrm{d} t} \bar{x}_{r}= & \left(A_{0}-\left(B+\widetilde{K}_{2}(\varepsilon)\right) \widetilde{K}_{1}^{-1}(\varepsilon) C_{m}\right) \bar{x}_{r} \\
& +\left(B+\widetilde{K}_{2}(\varepsilon)\right) \widetilde{K}_{1}^{-1}(\varepsilon) \bar{y} \\
u_{0}= & -\widetilde{K}_{1}^{-1}(\varepsilon) C_{m} \bar{x}_{r}+\widetilde{K}_{1}^{-1}(\varepsilon) \bar{y} .
\end{aligned}
$$

Inverting once again the right inverse approximation (A.2), we get after algebraic reductions (see Fact 1 of [5] or Lemma 3 of [8]):

$$
\frac{\mathrm{d}}{\mathrm{d} t} \bar{\zeta}=A_{0} \bar{\zeta}+\left(B+\widetilde{K}_{2}(\varepsilon)\right) u_{0}, \quad \bar{y}=C_{m} \bar{\zeta}+\widetilde{K}_{1}(\varepsilon) u_{0} .
$$

From (A.3) and (A.1), we finally get:

$$
\begin{aligned}
& \widetilde{K}_{1}(\varepsilon) u_{0}(\mathrm{~s})-\bar{y}(\mathrm{~s})= \\
= & -C_{m}\left(\mathrm{sI}_{n}-A_{0}\right)^{-1}\left(B+\widetilde{K}_{2}(\varepsilon)\right) u_{0}(\mathrm{~s}), \\
= & -C_{m} \sum_{i=1}^{n} A_{0}^{i-1}\left(B+\widetilde{K}_{2}(\varepsilon)\right) \mathrm{s}^{-i} u_{0}(\mathrm{~s}), \\
= & -C_{m}\left(\mathcal{C}_{\left(A_{0}, B\right)}+\mathcal{C}_{\left(A_{0}, \widetilde{K}(\varepsilon)\right)}\right) \Psi_{m}\left(\mathrm{~s}^{-1}\right) u_{0}(\mathrm{~s}), \\
= & -C_{m}\left(\mathrm{I}_{m}+\mathcal{C}_{\left(A_{0}, \widetilde{K}_{2}(\varepsilon)\right)} \mathcal{C}_{\left(A_{0}, B\right)}^{r}\right) x(\mathrm{~s}) .
\end{aligned}
$$

\section{B. Proof of Theorem 2}

During $t \in[0, \beta T)$ the quadrotor receives data from the SN and during $t \in[\beta T, T)$ it transmits the data to the DN. Let us denote the periodic trajectory of the quadrotor as $\mathbf{f}(t)=\mathbf{f}_{1}(t) \forall t \in[0, \beta T)$ and $\mathbf{f}(t)=\mathbf{f}_{2}(t) \forall t \in[\beta T, T)$ where $\mathbf{f}(t)=[\mathrm{x}(t) \mathrm{y}(t)]^{\mathrm{T}}$. Now, considering (4.4) we can write the initial and final conditions of $\mathbf{f}_{1}(t)$ and $\mathbf{f}_{2}(t)$ as $\mathbf{f}_{1}(0)=\left[0, \mathrm{y}_{0}\right]^{\mathrm{T}}, \mathbf{f}_{1}(\beta T)=\left[0, \mathrm{y}_{1}\right]^{\mathrm{T}}, \mathrm{d} \mathbf{f}_{1}(0) / \mathrm{d} t=$ $\left[\begin{array}{ll}v_{0}^{\mathrm{x}}, & v_{0}^{\mathrm{y}}\end{array}\right]^{\mathrm{T}}, \mathrm{d} \mathbf{f}_{1}(\beta T) / \mathrm{d} t=\left[\begin{array}{ll}v_{1}^{\mathrm{x}}, & v_{1}^{\mathrm{y}}\end{array}\right]^{\mathrm{T}}, \mathbf{f}_{2}(\beta T)=\left[\begin{array}{ll}0, & \mathrm{y}_{1}\end{array}\right]^{\mathrm{T}}$, $\mathbf{f}_{2}(T)=\left[0, \mathrm{y}_{0}\right]^{\mathrm{T}}, \mathrm{d} \mathbf{f}_{2}(\beta T) / \mathrm{d} t=\left[v_{1}^{\mathrm{x}}, v_{1}^{\mathrm{y}}\right]^{\mathrm{T}}$ and $\mathrm{d} \mathbf{f}_{2}(T) / \mathrm{d} t=$ $\left[v_{0}^{\mathrm{x}}, v_{0}^{\mathrm{y}}\right]^{\mathrm{T}}$.

Let us propose the following alternative optimization problem. During $t \in[0, \beta T)$ the quadrotor receives data from the $\mathrm{SN}$ with bit rate $R\left(\mathbf{p}(t), \mathbf{p}_{0}\right)$. During $t \in[\beta T, T]$ the quadrotor also receives data from the $\mathrm{DN}$ but with bit rate $R\left(\mathbf{p}(t), \mathbf{p}_{1}\right)$. The problem is to maximize the data received by the quadrotor during $t \in[0, T]$ with the constraint that the data received from the DN and the $\mathrm{SN}$ must be identical. And we also take into account the constraints (4.4) and the ones defined in (4.2), (4.3). This alternative optimization problem is equivalent to (4.2), (4.3) (i.e., they have the same solution). This problem can be written as two coupled optimization problems. The first one is:

$$
\max _{\bar{\theta}, \bar{\phi}, \beta} \int_{0}^{\beta T} \log _{2}\left(1+\frac{P / \sigma^{2}}{(D / 2-\mathrm{x}(t))^{2}+\mathrm{y}^{2}(t)}\right) \mathrm{d} t
$$

subject to the constraints

$$
\begin{aligned}
& (D / 2-\mathrm{x}(t))^{2}+\mathrm{y}^{2}(t) \geq d_{\text {min }}, \times(t) \geq 0, \forall t \in[0, \beta T] \\
& \mathrm{d}^{2} \times(t) / \mathrm{d} t^{2}=g \bar{\theta}, \mathrm{d}^{2} \mathrm{y}(t) / \mathrm{d} t^{2}=g \bar{\phi}, \\
& \mathrm{x}(0)=\mathrm{x}(\beta T)=0, \mathrm{y}(0)=\mathrm{y}_{0}, \mathrm{y}(\beta T)=\mathrm{y}_{1}, \\
& \mathrm{~d} \times(0) / \mathrm{d} t=v_{0}^{\mathrm{x}} \geq 0, \mathrm{dx}(\beta T) / \mathrm{d} t=v_{1}^{\mathrm{x}} \leq 0, \\
& \mathrm{dy}(0) / \mathrm{d} t=v_{0}^{\mathrm{y}}, \operatorname{dy}(\beta T) / \mathrm{d} t=v_{1}^{\mathrm{y}},
\end{aligned}
$$


and the second one:

$$
\max _{\bar{\theta}, \bar{\phi}, \beta} \int_{\beta T}^{T} \log _{2}\left(1+\frac{P / \sigma^{2}}{(D / 2+\mathrm{x}(t))^{2}+\mathrm{y}^{2}(t)}\right) \mathrm{d} t
$$

subject to the constraints

$$
\begin{aligned}
& (D / 2+\mathrm{x}(t))^{2}+\mathrm{y}^{2}(t) \geq d_{\text {min }} \mathrm{x}(t) \leq 0, \forall t \in[\beta T, T] \\
& \mathrm{d}^{2} \mathrm{x}(t) / \mathrm{d} t^{2}=g \bar{\theta}, \mathrm{d}^{2} \mathrm{y}(t) / \mathrm{d} t^{2}=g \bar{\phi}, \\
& \mathrm{x}(\beta T)=\mathrm{x}(T)=0, \mathrm{y}(\beta T)=\mathrm{y}_{1}, \mathrm{y}(T)=\mathrm{y}_{0}, \\
& \mathrm{dx}(\beta T) / \mathrm{d} t=v_{1}^{\mathrm{x}} \leq 0, \mathrm{dx}(T) / \mathrm{d} t=v_{0}^{\mathrm{x}} \geq 0, \\
& \mathrm{dy}(\beta T) / \mathrm{d} t=v_{1}^{\mathrm{y}}, \operatorname{dy}(T) / \mathrm{d} t=v_{0}^{\mathrm{y}},
\end{aligned}
$$

Both optimization problems are coupled by the initial and final conditions (i.e., $\mathrm{y}_{0}, \mathrm{y}_{1}, v_{0}^{\mathrm{x}}, v_{0}^{\mathrm{y}}, v_{1}^{\mathrm{x}}$ and $v_{1}^{\mathrm{y}}$ ) as well as by the fact that the quadrotor must receive the same amount of data from the $\mathrm{SN}$ and the $\mathrm{DN}$ :

$$
\int_{0}^{\beta T} R\left(\mathbf{p}(t), \mathbf{p}_{0}\right) \mathrm{d} t=\int_{\beta T}^{T} R\left(\mathbf{p}(t), \mathbf{p}_{1}\right) \mathrm{d} t
$$

Let us refer to the optimum trajectory solving (B.1), (B.2) as $\mathbf{g}_{1}^{*}(t ; 0, \beta T)=\left[\begin{array}{ll}\mathrm{x}_{1}^{*}(t) & \mathrm{y}_{1}^{*}(t)\end{array}\right]^{\mathrm{T}}$ which is defined over $t \in[0, \beta T]$ and with initial and final conditions:

$$
\begin{gathered}
\mathrm{x}_{1}^{*}(0)=0, \mathrm{y}_{1}^{*}(0)=\mathrm{y}_{0}, \mathrm{x}_{1}^{*}(\beta T)=0, \mathrm{y}_{1}^{*}(\beta T)=\mathrm{y}_{1}, \\
\mathrm{dx} \mathrm{x}_{1}^{*}(0) / \mathrm{d} t=v_{0}^{\mathrm{x}}, \mathrm{dy}_{1}^{*}(0) / \mathrm{d} t=v_{0}^{\mathrm{y}}, \\
\mathrm{d} \mathrm{x}_{1}^{*}(\beta T) / \mathrm{d} t=v_{1}^{\mathrm{x}}, \mathrm{dy}_{1}^{*}(\beta T) / \mathrm{d} t=v_{1}^{\mathrm{y}} .
\end{gathered}
$$

Then, let us refer to the optimum trajectory solving (B.3), (B.4) as $\mathbf{g}_{2}^{*}(t ; \beta T, T)=\left[\mathrm{x}_{2}^{*}(t), \mathrm{y}_{2}^{*}(t)\right]^{\mathrm{T}}$ which is defined over $t \in[\beta T, T]$ and with initial and final conditions:

$$
\begin{gathered}
\mathrm{x}_{2}^{*}(\beta T)=0, \mathrm{y}_{2}^{*}(\beta T)=\mathrm{y}_{1}, \quad \mathrm{x}_{2}^{*}(T)=0, \mathrm{y}_{2}^{*}(T)=\mathrm{y}_{0}, \\
\mathrm{~d} \mathrm{x}_{2}^{*}(\beta T) / \mathrm{d} t=v_{1}^{\mathrm{x}}, \quad \mathrm{dy}_{2}^{*}(\beta T) / \mathrm{d} t=v_{1}^{\mathrm{y}} \\
\mathrm{dx}_{2}^{*}(T) / \mathrm{d} t=v_{0}^{\mathrm{x}}, \quad \mathrm{dy}_{2}^{*}(T) / \mathrm{d} t=v_{0}^{\mathrm{y}} .
\end{gathered}
$$

Now, let us define the following trajectory for $t \in[\beta T, T]$ :

$$
\mathbf{g}_{3}^{*}(t ; \beta T, T) \triangleq \mathbf{g}_{2}^{*}((1+\beta) T-t ; \beta T, T) ;
$$

with initial and final conditions:

$$
\begin{gathered}
\mathrm{x}_{3}^{*}(\beta T)=\mathrm{x}_{3}^{*}(T)=0, \mathrm{y}_{3}^{*}(\beta T)=\mathrm{y}_{2}^{*}(T), \mathrm{y}_{3}^{*}(T)=\mathrm{y}_{2}^{*}(\beta T) \\
\mathrm{d} x_{3}^{*}(\beta T) / \mathrm{d} t=-\mathrm{d} \mathrm{x}_{2}^{*}(T) / \mathrm{d} t, \mathrm{dy}_{3}^{*}(\beta T) / \mathrm{d} t=\mathrm{dy}_{2}^{*}(T) / \mathrm{d} t \\
\mathrm{dx}_{3}^{*}(T) / \mathrm{d} t=-\mathrm{d} x_{2}^{*}(\beta T) / \mathrm{d} t, \mathrm{dy}_{3}^{*}(T) / \mathrm{d} t=-\mathrm{dy}_{2}^{*}(\beta T) / \mathrm{d} t
\end{gathered}
$$

So, if the trajectory $\mathbf{g}_{2}^{*}(t ; \beta T, T)$ is solution to (B.3), (B.4) then the trajectory $\mathbf{g}_{3}^{*}(t ; 0,(1-\beta) T)$, defined over $t \in[0,(1-\beta) T]$, is solution to the following optimization problem:

$$
\max _{\bar{\theta}, \bar{\phi}, \beta} \int_{0}^{(1-\beta) T} \log _{2}\left(1+\frac{P / \sigma^{2}}{(D / 2+\mathrm{x}(t))^{2}+\mathrm{y}^{2}(t)}\right) \mathrm{d} t
$$

subject to the following constraints

$$
\begin{aligned}
& (D / 2+\mathrm{x}(t))^{2}+\mathrm{y}^{2}(t) \geq d_{\text {min }} \mathrm{x}(t) \leq 0, \forall t \in[0,(1-\beta) T] \\
& \mathrm{d}^{2} \mathrm{x} / \mathrm{d} t^{2}=g \bar{\theta}, \mathrm{d}^{2} \mathrm{y}(t) / \mathrm{d} t^{2}=g \bar{\phi}, \\
& \mathrm{x}(0)=\mathrm{x}((1-\beta) T)=0, \mathrm{y}(0)=\mathrm{y}_{0}, \mathrm{y}((1-\beta) T)=\mathrm{y}_{1}, \\
& \mathrm{dx}(0) / \mathrm{d} t=-v_{0}^{\mathrm{x}} \geq 0, \mathrm{dx}((1-\beta) T) / \mathrm{d} t=-v_{1}^{\mathrm{x}} \leq 0, \\
& \mathrm{dy}(0) / \mathrm{d} t=-v_{0}^{\mathrm{y}}, \operatorname{dy}((1-\beta) T) / \mathrm{d} t=-v_{1}^{\mathrm{y}},
\end{aligned}
$$

Comparing (B.1), (B.2) with (B.8), (B.9) we observe that $\mathbf{g}_{3}^{*}(t+\beta T ; \beta T, T)$ and $\mathbf{g}_{1}^{*}(t ; 0, \beta T)$ are related as follows:

$$
\mathrm{x}_{3}^{*}(t+\beta T)=-\mathrm{x}_{1}^{*}\left(\frac{t(1-\beta)}{\beta}\right), \quad \mathrm{y}_{3}^{*}(t+\beta T)=\mathrm{y}_{1}^{*}\left(\frac{t(1-\beta)}{\beta}\right)
$$

Now, if:

$$
\mathrm{y}_{0}=-\mathrm{y}_{1}, \quad v_{0}^{\mathrm{x}}=-v_{1}^{\mathrm{x}}, \quad v_{0}^{\mathrm{y}}=-v_{1}^{\mathrm{y}},
$$

then, by combining (B.6), (B.7), (B.10) and (B.11), we get (4.5). So, if the relations described in (B.11) hold, then (4.5) also holds and then by applying this to (B.3), (B.4) and then comparing it to (B.1), (B.2) we note that the solution to the pair of optimization problems (B.1), (B.2) and (B.3), (B.4) (coupled by the initial and final conditions as well as by (B.5)) requires that both phases of the trajectory have the same duration and so the optimum $\beta^{*}=0.5$.

\section{REFERENCES}

[1] Armentano, V.A. (1986). The pencil $(s E-A)$ and controllabilityobservability for generalized linear systems: a geometric approach. SIAM Journal on Control and Optimization, 24(4), 616-638.

[2] Bernhard P. (1982). On singular implicit dynamical systems. SIAM Journal on Control and Optimization, 20(5), 612-633.

[3] Cook M.V. (2013). Flight Dynamics Principles. A Linear Systems Approach to Aircraft Stability and Control Elsevier Ltd., New York.

[4] G. H. Hardy. A Course of Pure Mathematics, Cambridge University Press. 1975.

[5] Bonilla, M. and Malabre, M. (1990). One side invertibility for implicit descriptions. 29th IEEE CDC, pp. 3601-3602.

[6] Bonilla, M., Malabre, M. and Fonseca, M. (1997). On the approximation of non-proper control laws. Int. J. Control, Vol. 68, No. 4, pp. 775-796.

[7] Bonilla, M. and Malabre, M. (2003). On the control of linear systems having internal variations. Automatica, Vol. 39, pp. 1989-1996.

[8] Bonilla, M., Figueroa, M., Malabre, M. and Martínez, J.C. (2007). Left invertibility and duality for linear systems. Linear Algebra and its Applications, Vol. 425, pp. 345-373.

[9] Blas, L.A., Bonilla, M., Malabre, M., Azhmyakov, V. and Salazar, S. (2017). IFAC-World Congress, pp. 2596-2601. July 9 - 14, 2017. Toulouse, France.

[10] Gantmacher, F.R. (1977). The Theory of Matrices. Vol. II, New York: Chelsea.

[11] Kailath, T. (1980). Linear Systems. Prentice-Hall, Inc. Englewood Cliffs, New Jersey.

[12] Wonham, W.M. (1985). Linear Multivariable Control: A Geometric Approach. Springer-Verlag, 3rd edition, New York.

[13] Goldsmith, A. (2005). Wireless Communications. Cambridge University Press, New York.

[14] D. E. Kirk (2004). Optimal control theory: An introduction.Dover Publications, Inc..

[15] S. Russell, P. Norving ( 2003). Artificial Intelligence: A Modern Approach, Prentice Hall.

[16] B. Baron, P. Spathis, M. Dias de Amorim , Y. Viniotis and M. H. Ammar. (2019) Mobility as an Alternative Communication Channel: A Survey. IEEE Communications Surveys \& Tutorials, 21(1), pp. 289 $-314$.

[17] U. Ali, H. Cai ; Y. Mostofi and Y. Wardi (2016). Motion and Communication Co-optimization with Path Planning and Online Channel Prediction. American Control Conference (ACC).

[18] J. Fink, A. Ribeiro and V. Kumar (2013) Robust Control of Mobility and Communications in Autonomous Robot Teams IEEE Access, vol. 1, pp. $290-309$.

[19] D. Bonilla Licea; E. Nurellari and M. Ghogho (2018). Energy Balancing for Robotic Aided Clustered Wireless Sensor Networks Using Mobility Diversity Algorithms 26th European Signal Processing Conference (EUSIPCO) . 